\title{
q-Path entropy phenomenology for phase-space curves
}

\author{
D. J. Zamora ${ }^{1}$ M. C. Rocca ${ }^{1}$, A. Plastino' ${ }^{1}$, and G. L. Ferri ${ }^{2}$ \\ ${ }^{1}$ La Plata National University and Argentina's National Research Council \\ (IFLP-CCT-CONICET)-C. C. 727, 1900 La Plata - Argentina \\ 2 Fac. de C. Exactas-National University La Pampa, \\ Peru y Uruguay, Santa Rosa, La Pampa, Argentina
}

June 14, 2021

\begin{abstract}
We describe the phenomenology of the classical q-path entropy of a phase-space curve. This allows one to disclose an entropic force-like mechanism that is able to mimic some phenomenological aspects of the strong force, such as confinement, hard core, and asymptotic freedom. Keywords: Phase-space curves; Entropic force; q-statistical mechanics
\end{abstract}




\section{Introduction}

\section{$1.1 \quad$ q-Statistics}

The so called q-statistical mechanics has been used in multiple applications in the last years $[1,2,3,4,5,6,7,8,9,10,11,12$, being of great relevance for astrophysics, particularly for self-gravitating systems [13, 16, 17, 26]. Additionally, it was useful in variegated scientific fields. It has originated several thousands of both papers and authors [2]. Research on its structural features is important for astronomy, physics, neurology, biology, economic sciences, etc. Its success reaffirms the idea that a great deal of physics derives from exclusively statistical considerations, rather than mechanical ones. A foremost example lies in its application to high energy physics. Here q-statistics seems to adequately describe the transverse momentum distributions of different hadrons [18, 19, 20]. In this sense, $\mathbf{q}=1.15$ has acquired particular relevance [18, 19, 20].

\subsection{Our goals}

We describe in this work the phenomenology of classical q-path entropies for arbitrary phase-space curves $\Gamma$, disclosing in such a way interesting features, like confinement and hard-cores. Of course, by confinement we are reminded of the phenomenon that impedes isolation of color charged particles (quarks that cannot be isolated singularly) and thus cannot be directly detected, while by asymptotic freedom we make reference to a property of some gauge theories that generates particles' bonds to become asymptotically weaker as distance diminishes, and at high momenta. Our curves-research will yield, surprisingly enough, a simple, classical entropic mechanism that is able to mimic some of above cited phenomena.

Remind that the entropic force is a phenomenological one that derives from statistical tendencies to entropic growth [21, 22, 23, 24, 25, 30, 27, 28, 29], without appealing to any specific underlying microscopic interaction. The foremost example is the elasticity of a freely-jointed polymer molecules [21, 22]. It is remarkable that Verlinde has argued that gravity can also be understood in terms of an entropic force [23].

Here we use q-path entropies to demonstrate that confinement can classically emerge from entropic forces, by appeal to a quadratic Hamiltonian in phase- 
space. These Hamiltonians are well know, classically and quantumly. For them, the correspondence between classical and quantum mechanics is exact. Unfortunately, explicit formulas are not trivial.

Knowledge of quadratic Hamiltonians is of utility for investigating more general Hamiltonians (and their associated Schroedinger equations) in a semiclassical scenario. Quadratic Hamiltonians are relevant in partial differential equations: they yield non trivial instances of wave propagation phenomena. Quadratic Hamiltonians also help in gaining insight into properties of more involved Hamiltonians used in quantum theory.

We thus will appeal to quadratic Hamiltonians in a classical environment so as to learn whether some interesting properties emerge concerning the entropic force along phase-space curves, which will indeed be the fact. A similar previous analysis involving the $\mathbf{q}=1$, ordinary Boltzmann entropy has been reported in [30]. $q \neq 1$ will be seen to add some interesting refinements.

The paper is organized as follows. Section 2 reviews basic notions. In Section 3 we compute the q-path entropy and associated mean energy for the harmonic oscillator (HO). Section 4 deals with the concomitant energyequipartition. Entropic forces linked to path entropies are the subject of Section 5. We specialize this force in Section 6. Conclusions are drawn in Section 7 .

\section{Preliminaries}

We consider a particle, moving in phase-space, attached to a harmonic oscillatorspring connected to the origin (the center of HO-attraction). We write the Hamiltonian in the fashion

$$
\mathrm{H}(\mathrm{P}, \mathrm{Q})=\mathrm{P}^{2}+\mathrm{Q}^{2}
$$

where both $\mathrm{P}^{2}$ and $\mathrm{Q}^{2}$ have the dimension of $\mathrm{H}$. Our environment is a Tsallisone [1] for which probability distributions are so-called q-exponentials, that read [1]

$$
e_{\mathrm{q}}(x)=[1+(1-q) x]^{1 /(1-q)} \text {. }
$$

The partition function in a Tsallis' scenario is defined as [1]

$$
Z(\beta)=\int_{-\infty}^{\infty} \int_{-\infty}^{\infty} e_{q}[-\beta H(P, Q)] d P d Q
$$


or

$$
Z(\beta)=\int_{-\infty}^{\infty} \int_{-\infty}^{\infty}[1+(q-1) \beta H(P, Q)]^{\frac{1}{1-q}} d P d Q,
$$

where $1 \leq \mathrm{q}<2$ is the non-extensibility parameter. Note that, if $\mathrm{q} \rightarrow 1$, the partition function reduces to the usual one, that means, the GibbsBoltzmann's partition function. Of course, $\beta$ is the inverse temperature. In a Jaynes' MaxEnt information-theory context [14, $\beta$ is a Lagrange multiplier that guarantees conservation of the mean energy, regarded as a priori datum, when one maximizes the entropy (in order to obtain the appropriate probability distribution). Jaynes' theory does not need neither ensembles nor heat baths [14].

Appealing now to the change of variables

$$
\mathrm{U}=\mathrm{P}^{2}+\mathrm{Q}^{2}, \mathrm{Q}^{\prime}=\sqrt{\mathrm{U}-\mathrm{P}^{2}}
$$

we obtain

$$
Z(\beta)=\pi \int_{0}^{\infty}[1+(q-1) \beta u]^{\frac{1}{1-q}} d u .
$$

Evaluating now (2.6) we have (see ref.[31])

$$
Z(\beta)=\frac{\pi}{\beta(q-1)} B\left[1, \frac{2-q}{q-1}\right],
$$

where $B[a, b]$ is the beta function. Thus,

$$
Z(\beta)=\frac{\pi}{\beta(q-1)} \frac{\Gamma(1) \Gamma\left(\frac{2-q}{q-1}\right)}{\Gamma\left(\frac{1}{q-1}\right)},
$$

or equivalently,

$$
Z(\beta)=\frac{\pi}{\beta(2-q)}
$$

If $q \rightarrow 1$, then

$$
\mathrm{Z} \rightarrow \frac{\pi}{\beta}
$$

and Eq. 2.9 thus reduces to the expression obtained in [30] for the GibbsBoltzmann statistics. Similarly, for the mean value of the energy we have

$$
<\mathrm{U}>(\beta)=\frac{1}{Z} \int_{-\infty}^{\infty} \int_{-\infty}^{\infty} \mathrm{H}(\mathrm{P}, \mathrm{Q})[1+(\mathrm{q}-1) \beta \mathrm{H}(\mathrm{P}, \mathrm{Q})]^{\frac{1}{1-q}} \mathrm{dPdQ}
$$


or

$$
<\mathrm{U}>(\beta)=\frac{\pi}{\mathrm{Z}} \int_{0}^{\infty} \mathrm{U}[1+(\mathrm{q}-1) \beta \mathrm{U}]^{\frac{1}{1-q}} \mathrm{du}
$$

The result of 2.12 is

$$
<\mathrm{U}>(\beta)=\frac{\pi}{\beta^{2}(\mathrm{q}-1)^{2} \mathrm{Z}} \mathrm{B}\left[2, \frac{3-2 \mathrm{q}}{\mathrm{q}-1}\right],
$$

that can be recast as

$$
<\mathrm{U}>(\beta)=\frac{\pi}{\beta^{2} Z} \frac{1}{(2-q)(3-2 q)} .
$$

Replacing above the value of $\mathbf{Z}$ we obtain

$$
<\mathrm{U}>(\beta)=\frac{1}{\beta(3-2 q)},
$$

with the restriction $1 \leq \mathrm{q}<1.5$ in order to guarantee the non-divergence of $<\mathrm{U}>$. When $\mathrm{q} \rightarrow 1$ we obtain

$$
<\mathrm{U}>\rightarrow \frac{1}{\beta},
$$

and for the entropy we have

$$
S(\beta)=\ln _{2-q} Z+Z^{q-1} \beta<u>,
$$

where $\ln _{\mathrm{q}}(z)$ is the $\mathrm{q}$-logarithm function defined as

$$
\ln _{\mathrm{q}}(z)=\frac{z^{1-\mathrm{q}}-1}{1-\mathrm{q}}
$$

Appropriate replacing in 2.17) we are led to

$$
S(\beta)=Z^{q-1}\left(\beta<U>+\frac{1}{q-1}\right)-\frac{1}{q-1},
$$

and

$$
S(\beta)=\left[\frac{\pi}{\beta(2-q)}\right]^{q-1} \frac{(2-q)}{(3-2 q)(q-1)}-\frac{1}{q-1} .
$$

This result for $S$ is valid for the interval $1<q<2$ and, of course, outside the poles, according to Ref. [31]. Remember that the integrations here involved yield finite result only for $1<\mathrm{q}<2$, and otherwise diverge. 


\section{Path entropy}

We focus now on the concept of path entropy [30]. The path is a phase-space curve $\Gamma$ parameterized by the variable Q. Following the procedure of [30], we shall specialize equations 2.6 and 2.12 to curves $\Gamma$. We first define

$$
\mathrm{Z}(\beta, \Gamma)=\pi \int_{\Gamma}[1+(\mathrm{q}-1) \beta \mathrm{U}(\mathrm{P}, \mathrm{Q})]^{\frac{1}{1-q}} \mathrm{dU}(\mathrm{P}, \mathrm{Q})
$$

If we consider curves (parametrized by the independent variable $Q$ ) passing through the origin, we have $\mathrm{P}(0)=0$ and $\mathrm{Q}=0$, and consequently $\mathrm{U}(0,0)=$ 0 . Since the integrand in equation 3.1 is an exact differential and the integral only depends on the end point $Q_{0}$ we have

$$
\mathrm{Z}\left(\beta, \mathrm{Q}_{0}\right)=\pi \int_{0}^{\mathrm{U}\left(\mathrm{Q}_{0}, \mathrm{P}\left(\mathrm{Q}_{0}\right)\right)}[1+(\mathrm{q}-1) \beta \mathrm{U}(\mathrm{P}, \mathrm{Q})]^{\frac{1}{1-q}} \mathrm{du}(\mathrm{P}, \mathrm{Q}),
$$

or

$$
\mathrm{Z}\left(\beta, \mathrm{Q}_{0}\right)=\frac{\pi}{(2-\mathrm{q}) \beta}\left\{1-\left[1+(\mathrm{q}-1) \beta \mathrm{U}\left(\mathrm{P}\left(\mathrm{Q}_{0}\right), \mathrm{Q}_{0}\right)\right]^{\frac{2-q}{1-q}}\right\} .
$$

If $Q_{0} \rightarrow \infty$ then $Z\left(\beta, Q_{0}\right)$ reduces to expression 2.9. Moreover if $q \rightarrow 1$, then

$$
\mathrm{Z}\left(\beta, \mathrm{Q}_{0}\right) \rightarrow \frac{\pi}{\beta}\left(1-\mathrm{e}^{-\beta \mathrm{U}\left(\mathrm{P}\left(\mathrm{Q}_{0}\right), \mathrm{Q}_{0}\right)}\right) .
$$

In the same way, we have for the mean value of energy

$$
<\mathrm{U}>(\beta, \Gamma)=\frac{\pi}{\mathrm{Z}(\beta, \Gamma)} \int_{\Gamma} \mathrm{U}(\mathrm{P}, \mathrm{Q})[1+(\mathrm{q}-1) \beta \mathrm{U}(\mathrm{P}, \mathrm{Q})]^{\frac{1}{1-q}} \mathrm{dU}(\mathrm{P}, \mathrm{Q})
$$

or equivalently,

$$
<\mathrm{U}>\left(\beta, \mathrm{Q}_{0}\right)=\frac{\pi}{\mathrm{Z}\left(\beta, \mathrm{Q}_{0}\right)} \int_{0}^{\mathrm{U}\left(\mathrm{Q}_{0} \mathrm{P}\left(\mathrm{Q}_{0}\right)\right)} \mathrm{U}(\mathrm{P}, \mathrm{Q})[1+(\mathrm{q}-1) \beta \mathrm{U}(\mathrm{P}, \mathrm{Q})]^{\frac{1}{1-q}} d \mathrm{U}(\mathrm{P}, \mathrm{Q})
$$

If we evaluate 3.6 we obtain

$$
\begin{aligned}
<\mathrm{U}>\left(\beta, \mathrm{Q}_{0}\right)=\frac{\pi}{\mathrm{Z}\left(\beta, \mathrm{Q}_{0}\right) \beta^{2}}\left\{\frac{1-\left[1+(\mathrm{q}-1) \beta \mathrm{U}\left(\mathrm{P}\left(\mathrm{Q}_{0}\right), \mathrm{Q}_{0}\right)\right]^{\frac{3-2 \mathrm{q}}{1-q}}}{(3-2 \mathrm{q})(\mathrm{q}-1)}-\right. \\
\left.\frac{1-\left[1+(\mathrm{q}-1) \beta \mathrm{U}\left(\mathrm{P}\left(\mathrm{Q}_{0}\right), \mathrm{Q}_{0}\right)\right]^{\frac{2-q}{1-q}}}{(2-\mathrm{q})(1-\mathrm{q})}\right\}
\end{aligned}
$$


and, simplifying the last expression,

$$
<\mathrm{U}>\left(\beta, \mathrm{Q}_{0}\right)=\frac{1}{\beta(\mathrm{q}-1)}\left\{-1+\frac{(2-q)}{(3-2 \mathrm{q})} \frac{\left\{1-\left[1+(\mathrm{q}-1) \beta \mathrm{U}\left(\mathrm{P}\left(\mathrm{Q}_{0}\right), \mathrm{Q}_{0}\right)\right]^{\frac{3-2 \mathrm{q}}{1-q}}\right\}}{\left\{1-\left[1+(\mathrm{q}-1) \beta \mathrm{U}\left(\mathrm{P}\left(\mathrm{Q}_{0}\right), \mathrm{Q}_{0}\right)\right]^{\frac{2-q}{1-q}}\right\}}\right\} .
$$

If $\mathrm{Q}_{0} \rightarrow \infty$, then $<\mathrm{U}>\left(\beta, \mathrm{Q}_{0}\right)$ reduces to expression 2.15 . If $\mathrm{q} \rightarrow 1$, then

$$
<\mathrm{U}>\left(\beta, \mathrm{Q}_{0}\right) \rightarrow \frac{1-(1+\beta \mathrm{U}) e^{-\beta \mathrm{U}}}{\left(1-\mathrm{e}^{-\beta \mathrm{U}}\right) \beta}
$$

which is consistent with the results obtained in [30. Again, we can express the entropy via the partition function and the mean value of energy, i.e.,

$$
S\left(\beta, Q_{0}\right)=\ln _{2-q} Z\left(\beta, Q_{0}\right)+Z\left(\beta, Q_{0}\right)^{q-1} \beta<U>\left(\beta, Q_{0}\right),
$$

or, equivalently,

$$
\begin{gathered}
S\left(\beta, Q_{0}\right)=\frac{1}{q-1}\left\{\frac { ( 2 - q ) } { ( 3 - 2 q ) } [ \frac { \pi } { ( 2 - q ) \beta } ] ^ { ( q - 1 ) } \left\{1-\left[1+(q-1) \beta U\left(P\left(Q_{0}\right), Q_{0}\right)\right]^{\left.\frac{2-q}{1-q}\right\}^{(q-2)}}\right.\right. \\
\left.\left\{1-\left[1+(q-1) \beta U\left(P\left(Q_{0}\right), Q_{0}\right)\right]^{\frac{3-2 q}{1-q}}\right\}-1\right\} \cdot
\end{gathered}
$$

Note here that, for this $\mathrm{S}$-expression, the same considerations made above for Eq. 2.20 also hold. This is, the result for $S$ is valid for the interval $1<\mathrm{q}<2$ and, of course, outside the poles, according to Ref. [31]. We reiterate that the integrations here involved yield finite result only for $1<\mathrm{q}<2$, and otherwise diverge.

\section{Equipartition}

We find that

$$
<\mathrm{Q}^{2}>=\frac{1}{\mathrm{Z}} \int_{-\infty}^{\infty} \int_{-\infty}^{\infty} \mathrm{Q}^{2}\left[1+(\mathrm{q}-1) \beta\left(\mathrm{P}^{2}+\mathrm{Q}^{2}\right)\right]^{\frac{1}{1-q}} \mathrm{dPdQ}
$$

or

$$
<\mathrm{Q}^{2}>=\frac{\pi}{2 \mathrm{Z}} \int_{0}^{\infty} \mathrm{U}[1+(\mathrm{q}-1) \beta \mathrm{U}]^{\frac{1}{1-\mathrm{q}}} \mathrm{dU}
$$


while along the curve $\Gamma$,

$$
<\mathrm{Q}^{2}>(\beta, \Gamma)=\frac{\pi}{2 \mathrm{Z}(\beta, \Gamma)} \int_{\Gamma} \mathrm{U}[1+(\mathrm{q}-1) \beta \mathrm{U}]^{\frac{1}{1-q}} \mathrm{du}
$$

Thus,

$$
<\mathrm{Q}^{2}>\left(\beta, \mathrm{Q}_{0}\right)=\frac{\pi}{2 \mathrm{Z}\left(\beta, \mathrm{Q}_{0}\right)} \int_{0}^{\mathrm{u}\left(\mathrm{Q}_{0}, \mathrm{P}\left(\mathrm{Q}_{0}\right)\right)} \mathrm{U}[1+(\mathrm{q}-1) \beta \mathrm{U}]^{\frac{1}{1-q}} \mathrm{dU}
$$

This integral yields

$$
\begin{gathered}
<\mathrm{Q}^{2}>\left(\beta, \mathrm{Q}_{0}\right)=\frac{\pi}{2 \mathrm{Z}\left(\beta, \mathrm{Q}_{0}\right) \beta^{2}}\left\{\frac{1-\left[1+(\mathrm{q}-1) \beta \mathrm{U}\left(\mathrm{P}\left(\mathrm{Q}_{0}\right), \mathrm{Q}_{0}\right)\right]^{\frac{3-2 \mathrm{q}}{1-q}}}{(3-2 \mathrm{q})(\mathrm{q}-1)}-\right. \\
\left.\frac{1-\left[1+(\mathrm{q}-1) \beta \mathrm{U}\left(\mathrm{P}\left(\mathrm{Q}_{0}\right), \mathrm{Q}_{0}\right)\right]^{\frac{2-q}{1-q}}}{(2-\mathrm{q})(1-\mathrm{q})}\right\},
\end{gathered}
$$

and then

$$
<\mathrm{Q}^{2}>\left(\beta, \mathrm{Q}_{0}\right)=\frac{<\mathrm{U}>\left(\beta, \mathrm{Q}_{0}\right)}{2}
$$

that is,

$$
<\mathrm{Q}^{2}>\left(\beta, \mathrm{Q}_{0}\right)=<\mathrm{P}^{2}>\left(\beta, \mathrm{Q}_{0}\right)=\frac{<\mathrm{U}>\left(\beta, \mathrm{Q}_{0}\right)}{2},
$$

which, for $\mathrm{Q}_{0} \rightarrow \infty$ gives

$$
<\mathrm{Q}^{2}>=<\mathrm{P}^{2}>=\frac{<\mathrm{U}>}{2}=\frac{1}{2(3-2 \mathrm{q}) \beta},
$$

the q-equipartition recipe [15]. In this phenomenological environment, it obviously sets an upper bound for $q$, namely, $q<3 / 2$, that applies only to the q-path entropy and not at all to the wide world of q-non additivity.

\section{Entropic force}

According to [23, 24], the entropic force $F_{e}$ is given by

$$
F_{e}=\frac{1}{\beta} \frac{\partial S}{\partial Q_{0}}
$$


Since the end-point of the curve $Q_{0}$ is arbitrary, we may call it $Q$. In our case this is

$$
\mathrm{F}_{e}=\frac{\mathrm{Z}^{\mathrm{q}-2}}{\beta} \frac{\partial \mathrm{Z}}{\partial \mathrm{Q}}+(\mathrm{q}-1) \mathrm{Z}^{\mathrm{q}-2} \frac{\partial \mathrm{Z}}{\partial \mathrm{Q}}<\mathrm{U}>+\mathrm{Z}^{\mathrm{q}-1} \frac{\partial<\mathrm{U}>}{\partial \mathrm{Q}} .
$$

See that $\mathbf{Z}$ and $<\mathrm{U}>$ were already determined in 3.3 and 3.8 , respectively. Note that these quantities are functions of $\beta$ and $Q$. Recall that when we write $\mathrm{U}$ we refer to $\mathrm{U}(\mathrm{P}(\mathrm{Q}), \mathrm{Q})$. Accordingly, we have

$$
\frac{\partial Z}{\partial Q}=\pi \frac{\partial U}{\partial Q}[1+(q-1) \beta U]^{\frac{1}{1-q}}=2 \pi Q[1+(q-1) \beta U]^{\frac{1}{1-q}}
$$

and

$$
\begin{aligned}
\frac{\partial<U>}{\partial Q}= & \frac{(2-q)}{(3-2 q)(q-1)} \cdot \frac{2 Q}{\left\{1-[1+(q-1) \beta U]^{\frac{2-q}{1-q}}\right\}}\left\{(3-2 q)[1+(q-1) \beta U]^{\frac{2-q}{1-q}}\right. \\
& \left.-(2-q)[1+(q-1) \beta U]^{\frac{1}{1-q}} \frac{\left\{1-[1+(q-1) \beta U]^{\frac{3-2 q}{1-q}}\right\}}{\left\{1-[1+(q-1) \beta U]^{\frac{2-q}{1-q}}\right\}}\right\} \cdot(5.4)
\end{aligned}
$$

Rearranging terms and properly adding or subtracting when necessary, one we finds

$$
\begin{aligned}
& \frac{\partial<\mathrm{U}>}{\partial Q}=\frac{2 \mathrm{Q}(2-\mathrm{q})}{(\mathrm{q}-1)} \cdot \frac{[1+(q-1) \beta \mathrm{U}]^{\frac{1}{1-q}}}{\left\{1-[1+(q-1) \beta \mathrm{U}]^{\frac{2-q}{1-q}}\right\}} \\
& \left\{[1+(q-1) \beta \mathrm{U}]-1+1-\frac{(2-q)}{(3-2 q)} \frac{\left\{1-[1+(q-1) \beta U]^{\frac{3-2 q}{1-q}}\right\}}{\left\{1-[1+(q-1) \beta U]^{\frac{2-q}{1-q}}\right\}}\right\},
\end{aligned}
$$

while (5.5) can be written as

$$
\frac{\partial<U>}{\partial Q}=2 Q(2-q) \frac{[1+(q-1) \beta U]^{\frac{1}{1-q}}}{\left\{1-[1+(q-1) \beta U]^{\frac{2-q}{1-q}}\right\}}\{\beta U-\beta<U>\}
$$


Thus, we have for $F_{e}$ the expression

$$
\begin{gathered}
F_{e}=\left(\frac{\pi}{\beta}\right)^{q-1} 2 Q(2-q)^{2-q} \frac{[1+(q-1) \beta U]^{\frac{1}{1-q}}}{\left(1-[1+(q-1) \beta U]^{\frac{2-q}{1-q}}\right)^{2-q}}\left\{\beta U+\frac{1}{q-1}\right. \\
\left.-\frac{1}{(q-1)} \frac{(2-q)^{2}}{(3-2 q)} \frac{\left\{1-[1+(q-1) \beta U]^{\frac{3-2 q}{1-q}}\right\}}{\left\{1-[1+(q-1) \beta U]^{\frac{2-q}{1-q}}\right\}}\right\} .
\end{gathered}
$$

This is of the form

$$
\mathrm{F}_{e}=\mathrm{K}(\mathrm{q}, \beta, \mathrm{U}[\mathrm{Q}, \mathrm{P}]) \mathrm{Q},
$$

and will be either attractive or repulsive. The entropic force is here "harmonic"like too. It is not conservative because it depends on $\mathrm{P}$ and not only on position. In the examples we have considered in this paper, $\mathrm{K}$ is positive. Where does it come from? We might argue as follows: we want our particle to traverse a given path in phase space. Such path may connect points corresponding to different $\mathrm{HO}$-energies. As a response to our traveling particle, a non conservative force arises that opposes its motion, in à la Chatelier fashion.

If $\mathrm{q} \rightarrow 1$,

$$
\begin{gathered}
\frac{\partial \mathrm{Z}}{\partial \mathrm{Q}} \rightarrow 2 \pi \mathrm{Q} e^{-\beta \mathrm{U}}, \\
\frac{\partial<\mathrm{U}>}{\partial \mathrm{Q}} \rightarrow \frac{2 \mathrm{Q} e^{-\beta \mathrm{U}}}{1-e^{-\beta \mathrm{U}}}\left[\frac{\beta \mathrm{U}}{1-e^{-\beta \mathrm{U}}}-1\right],
\end{gathered}
$$

and

$$
\mathrm{F}_{e} \rightarrow 2 \mathrm{Q} \beta \mathrm{U} \frac{e^{-\beta \mathrm{U}}}{\left(1-e^{-\beta \mathrm{U}}\right)^{2}} .
$$

Clearly, $\mathrm{K}$ is positive and the force repulsive. For $\mathrm{q}$ close to unity, a first order approximation yields

$$
\begin{gathered}
\mathrm{Z} \approx \pi \mathrm{U}, \\
\frac{\partial \mathrm{Z}}{\partial \mathrm{Q}} \approx 2 \pi \mathrm{Q}(1-\beta \mathrm{U}) \approx 2 \pi \mathrm{Q},
\end{gathered}
$$




$$
\begin{gathered}
<\mathrm{U}>\approx 0 \\
\frac{\partial<\mathrm{U}>}{\partial \mathrm{Q}} \approx 2 \mathrm{Q}(1-\beta \mathrm{U}) \approx 2 \mathrm{Q}
\end{gathered}
$$

and

$$
\mathrm{F}_{e} \approx \frac{\pi}{\beta}(\pi \mathrm{U})^{\mathrm{q}-2} 2 \mathrm{Q}+(\pi \mathrm{U})^{\mathrm{q}-1} 2 \mathrm{Q} \approx(\pi \mathrm{U})^{\mathrm{q}-1} 2 \mathrm{Q}\left(\frac{1}{\beta \mathrm{U}}+1\right) .
$$

Finally, the entropic force simplifies to

$$
\mathrm{F}_{\mathrm{e}} \approx(\pi \mathrm{U})^{\mathrm{q}-1} \frac{2 \mathrm{Q}}{\beta \mathrm{U}},
$$

with $\mathrm{K}$ positive and, for $\mathrm{q} \rightarrow 1$,

$$
\mathrm{F}_{\mathrm{e}} \approx \frac{2 \mathrm{Q}}{\beta \mathrm{U}},
$$

in agreement with [30]. We have carefully explored the whole $(q, p)$ plane and discovered that effects of $F_{e}$ are noticeable only in a small region of it.

We can still replace $\mathrm{U}$ in the equations above using $\mathrm{U}=\mathrm{P}^{2}+\mathrm{Q}^{2}$. One has

$$
\begin{gathered}
F_{e}=\left(\frac{\pi}{\beta}\right)^{q-1} 2 Q(2-q)^{2-q} \frac{\left[1+(q-1) \beta\left(P^{2}+Q^{2}\right)\right]^{\frac{1}{1-q}}}{\left(1-\left[1+(q-1) \beta\left(P^{2}+Q^{2}\right)\right]^{\frac{2-q}{1-q}}\right)^{2-q}} \\
\left\{\beta\left(P^{2}+Q^{2}\right)+\frac{1}{q-1}\right. \\
\left.-\frac{1}{(q-1)} \frac{(2-q)^{2}}{(3-2 q)} \frac{\left\{1-\left[1+(q-1) \beta\left(P^{2}+Q^{2}\right)\right]^{\frac{3-2 q}{1-q}}\right\}}{\left\{1-\left[1+(q-1) \beta\left(P^{2}+Q^{2}\right)\right]^{\frac{2-q}{1-q}}\right\}}\right\} .
\end{gathered}
$$

We understand by force's confinement "that there is just a region where the force is significant". We see that the entropic force is in this sense confined to just a small region of phase space. This confinement effect i) grows with $\mathrm{q}$ and ii) leads to asymptotic freedom (zero force) outside such a region. We note also that is the particle lies inside the potential-barrier, it will be hard for it to escape (no tunneling in classical physics). The peak of the entropic force becomes more pronounced as $\mathrm{q}$ increases. We point out that, as the kinetic energy, associated to the momentum, grows, so does confinement. These observations are illustrated by Figs. 1 and 2 . 


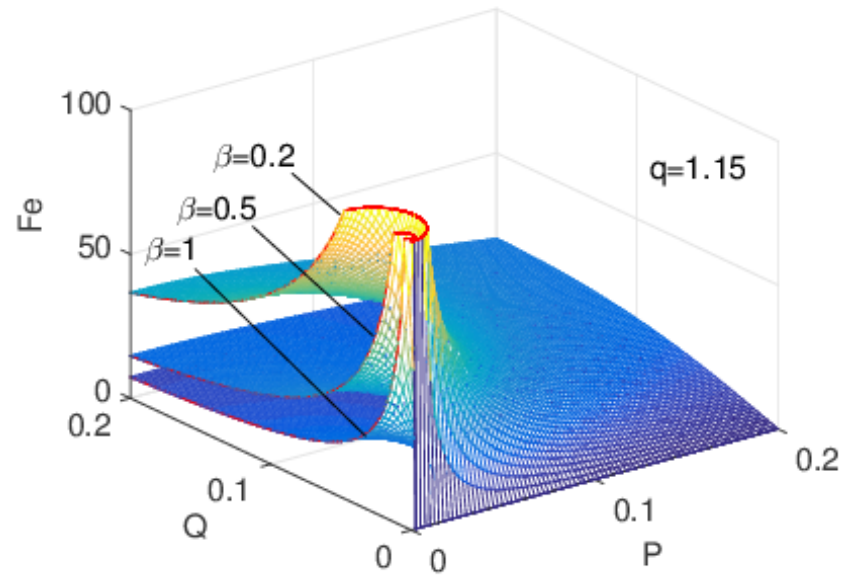

Figure 1: Behavior of the entropic force with T. We note that the entropic force grows with the temperature, as one should expect.

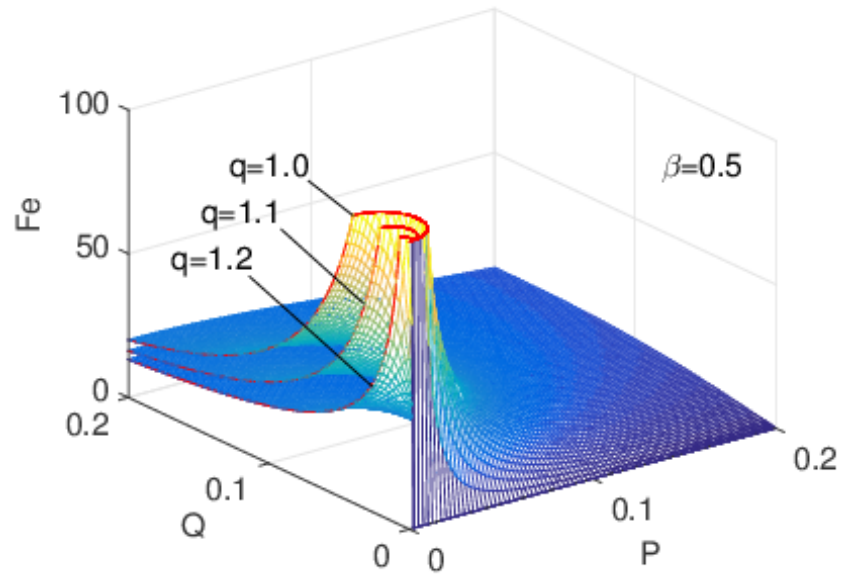

Figure 2: Behavior of the entropic force with $\mathbf{q}$. We see that the entropic force diminishes as q grows, which constitutes a new result. 


\section{The effects of the $\mathrm{HO}$ well}

Obviously, our particle also feels an harmonic force. Let us add its effect to that of $F_{e}$. We have

$$
\mathrm{F}_{\mathrm{HO}}=-\frac{1}{2} \frac{\partial<\mathrm{Q}^{2}>}{\partial \mathrm{Q}}=-\frac{1}{4} \frac{\partial<\mathrm{U}>}{\partial \mathrm{Q}} .
$$

In our case this becomes

$$
F_{H O}=-\frac{Q}{2}(2-q) \frac{[1+(q-1) \beta U]^{\frac{1}{1-q}}}{\left\{1-[1+(q-1) \beta U]^{\frac{2-q}{1-q}}\right\}}\{\beta U-\beta<U>\} .
$$

Note that, for $\mathrm{q} \rightarrow 1$,

$$
\mathrm{F}_{\mathrm{HO}} \rightarrow-\frac{\mathrm{Q}}{2} \frac{e^{-\beta \mathrm{U}}}{\left(1-e^{-\beta \mathrm{U}}\right)}\left(\frac{\beta \mathrm{U}}{1-e^{-\beta \mathrm{U}}}-1\right)
$$

or

$$
\mathrm{F}_{\mathrm{HO}} \rightarrow \frac{\mathrm{Q}}{2} \frac{e^{-\beta \mathrm{U}}}{\left(1-e^{-\beta \mathrm{U}}\right)^{2}}\left(1-\beta \mathrm{U}-e^{-\beta \mathrm{U}}\right) .
$$

The total force that the particle feels is

$$
\mathrm{F}_{\mathrm{T}}=\mathrm{F}_{\mathrm{e}}+\mathrm{F}_{\mathrm{HO}}=\mathrm{Z}^{\mathrm{q}-2} \frac{\partial \mathrm{Z}}{\partial \mathrm{Q}}\left(\frac{1}{\beta}+(\mathrm{q}-1)<\mathrm{U}>\right)+\frac{\partial<\mathrm{U}>}{\partial \mathrm{Q}}\left(\mathrm{Z}^{\mathrm{q}-1}-\frac{1}{4}\right),
$$


and then:

$$
\begin{gathered}
F_{T}=\left(\frac{\pi}{\beta}\right)^{q-1} 2 Q(2-q)^{2-q} \frac{\left[1+(q-1) \beta\left(P^{2}+Q^{2}\right)\right]^{\frac{1}{1-q}}}{\left(1-\left[1+(q-1) \beta\left(P^{2}+Q^{2}\right)\right]^{\frac{2-q}{1-q}}\right)^{2-q}} \\
\left\{\beta\left(P^{2}+Q^{2}\right)+\frac{1}{q-1}\right. \\
\left.-\frac{1}{(q-1)} \frac{(2-q)^{2}}{(3-2 q)} \frac{\left\{1-\left[1+(q-1) \beta\left(P^{2}+Q^{2}\right)\right]^{\frac{3-2 q}{1-q}}\right\}}{\left\{1-\left[1+(q-1) \beta\left(P^{2}+Q^{2}\right)\right]^{\frac{2-q}{1-q}}\right\}}\right\}- \\
\frac{Q}{2}(2-q) \frac{\left[1+(q-1) \beta\left(P^{2}+Q^{2}\right)\right]^{\frac{1}{1-q}}}{\left\{1-\left[1+(q-1) \beta\left(P^{2}+Q^{2}\right)\right]^{\frac{2-q}{1-q}}\right\}} \\
\left.\beta\left(P^{2}+Q^{2}\right)+\frac{1}{(q-1)}\left(1-\frac{(2-q)\left\{1-\left[1+(q-1) \beta\left(P^{2}+Q^{2}\right)\right]^{\frac{3-2 q}{1-q}}\right\}}{(3-2 q)} \frac{\left\{1-\left[1+(q-1) \beta\left(P^{2}+Q^{2}\right)\right]^{\frac{2-q}{1-q}}\right\}}{\{1}\right)\right]
\end{gathered}
$$

In order to appreciate the meaning of the above equation, consider its limit for $\mathrm{q} \rightarrow 1$,

$$
\mathrm{F}_{\mathrm{T}} \rightarrow \frac{\mathrm{Q}}{2} \frac{\mathrm{e}^{-\beta \mathrm{U}}}{\left(1-\mathrm{e}^{-\beta \mathrm{U}}\right)^{2}}\left(1+3 \beta \mathrm{U}-\mathrm{e}^{-\beta \mathrm{U}}\right)
$$

The absolute value of the force grows linearly with the distance Q. It also grows with $\mathrm{T}$.

Notice that, as above, the total force is confined (different from zero) to just a small region of phase space. Such confinement effect i) grows with q and ii) leads to asymptotic freedom (zero total force) outside such a region. Once again, the peak of the total force becomes more pronounced as $q$ increases. Also, as $F_{\text {total }}$ grows, so does confinement. We see that, here, the entropic force is a manifestation of the entropic change along a curve in phase space. As our particle traverses this path, it feels the action of $F_{e}$. These considerations are illustrated by Figs. 3 and 4 . 


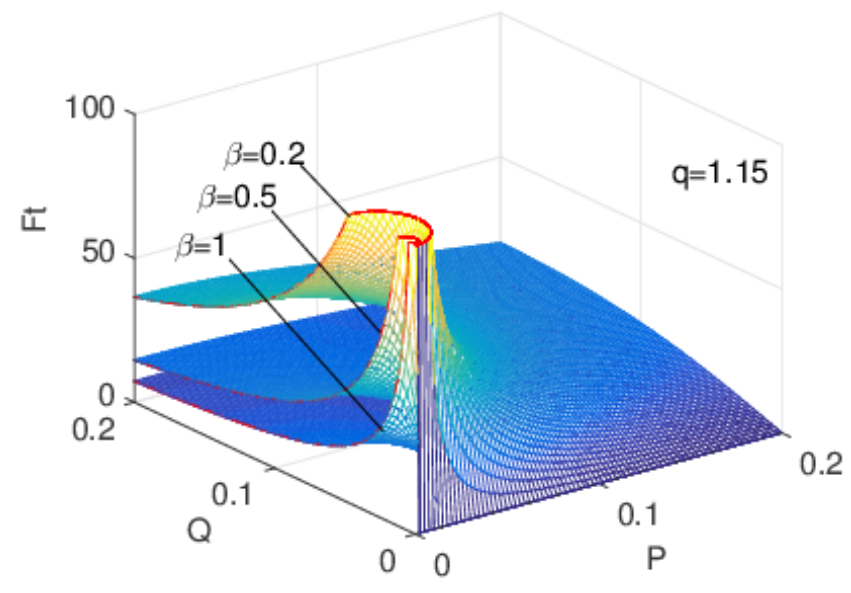

Figure 3: Total force associated to the path entropy as a function of $\mathrm{T}$. It grows as the temperature increases, as one should expect.

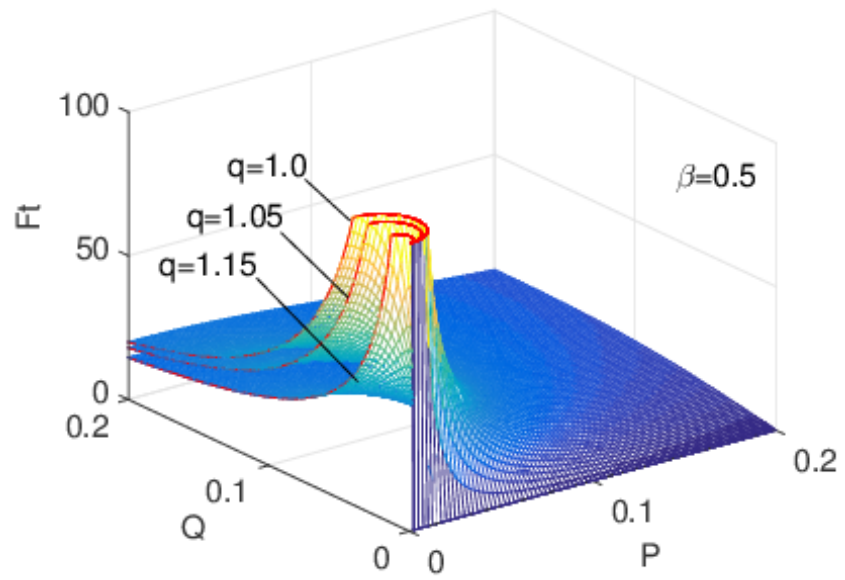

Figure 4: Total force associated to the path entropy as a function of $\mathrm{q}$. It diminishes as $\mathbf{q}$ grows. 


\section{Conclusions}

In this paper we have introduced the notion of classical q-path entropy and described its behavior for the classical harmonic oscillator (HO). In other words, we have described the phenomenology of the q-path entropy for the classical HO. One main facet of this phenomenology is the existence of qentropic force effects. We discovered that one can classically mimic, with the q-entropic force, aspects of another phenomenology: that of strong forces.

- Although we discussed arbitrary phase space curves $\Gamma$, in general our effects do not depended upon the specific $\Gamma$ chosen. Our q-phenomenology appears reasonable because a q-equipartition theorem holds.

- We discovered that the q-entropic force diverges at specific small areas of phase space (hard-core effect), vanishing outside those areas (confinement plus asymptotic freedom). The size of these areas is q-dependent. The "hardness" of the core also depends upon q. Adding a harmonic well does not modify this phenomenology.

- Also, the entropic force grows with q, an interesting result. The more non-additive the environment becomes, the stronger the entropic force. This force also increases with temperature, which constitutes a reasonable result.

- The q-dependence that we have just described justifies the appeal to a non-additive environment that is the leite motif of this effort. 


\section{References}

[1] M. Gell-Mann and C. Tsallis, Eds. Nonextensive Entropy: Interdisciplinary applications, Oxford University Press, Oxford, 2004.

[2] See http://tsallis.cat.cbpf.br/biblio.htm for a regularly updated bibliography on the subject.

[3] P-Jizba, J. Korbel, V. Zatloukal, Phys. Rev. E 95, 022103 (2017); L. S. F. Olavo, Phys. Rev. E 64, 036125 (2001).

[4] I. S. Oliveira: Eur. Phys. J. B 14, 43 (2000)

[5] E. K. Lenzi , R. S. Mendes: Eur. Phys. J. B 21, 401 (2001)

[6] C. Tsallis: Eur. Phys. J. A 40, 257 (2009)

[7] P. H. Chavanis: Eur. Phys. J. B 53, 487 (2006)

[8] G. Ruiz , C. Tsallis: Eur. Phys. J. B 67, 577 (2009)

[9] P. H. Chavanis , A. Campa: Eur. Phys. J. B 76, 581 (2010)

[10] N. Kalogeropoulos: Eur. Phys. J. B 87, 56 (2014)

[11] N. Kalogeropoulos: Eur. Phys. J. B 87, 138 (2014)

[12] A. Kononovicius, J. Ruseckas: Eur. Phys. J. B 87, 169 (2014)

[13] A. R. Plastino, A. Plastino, Phys. Lett. A 174, 384 (1993)

[14] E.T. Jaynes, Phys. Rev. 106 (1957) 620; 118 (1961) 171; Papers on probability, statistics and statistical physics, edited by R. D. Rosenkrantz, Reidel, Dordrecht, Holland, 1983; L. Brillouin, Science and Information Theory, Academic Press, New York (1956); WT Grandy, Jr., and PW Milonni, Physics and probability: Essays in honor of E.T. Jaynes, Cambridge University Press, Cambridge, England, 1993.

[15] A. R. Plastino, A. Plastino, C. Tsallis, Journal of Physics A 27 (1994) 5707.

[16] P. H. Chavanis, C. Sire, Physica A 356 (2005) 419; P.-H. Chavanis, J. Sommeria, Mon. Not. R. Astron. Soc. 296, 569 (1998). 
[17] D. Lynden-Bell, R. M. Lynden-Bell, Mon. Not. R. Astron. Soc. 181, 405 (1977).

[18] C. Tsallis, Introduction to Nonextensive Statistical Mechanics, Springer, Berlin, 2009.

[19] F. Barile et al. (ALICE Collaboration), EPJ Web Conferences 60, 13012 (2013); B. Abelev et al. (ALICE Collaboration), Phys. Rev. Lett. 111, 222301 (2013); Yu. V.Kharlov (ALICE Collaboration), Physics of Atomic Nuclei 76, 1497 (2013); ALICE Collaboration, Phys. Rev. C 91, (2015) ATLAS Collaboration, New J. Physics 13, 024609 (2011); 053033; CMS Collaboration, J. High Energy Phys. 05,064 (2011); CMS Collaboration, Eur. Phys. J. C 74, 2847 (2014)

[20] A. Adare et al (PHENIX Collaboration), Phys. Rev. D 83, 052004 (2011); PHENIX Collaboration, Phys. Rev. C 83, 024909 (2011); PHENIX Collaboration, Phys. Rev. C 83, 064903 (2011); PHENIX Collaboration, Phys. Rev. C 84, 044902 (2011)

[21] H.W. de Haan, G.W. Slater, Phys. Rev. E 87, 042604 (2013)

[22] M.F. Maghrebi, Y. Kantor, M. Kardar, Phys. Rev. E 86, 061801 (2012)

[23] E. Verlinde: J. High Energy Physics. 04, 29 (2011)

[24] A.D. Wissner-Gross, C.E. Freer, Phys. Rev. Lett. 110, 168702 (2013)

[25] R.C. Dewar, Entropy 11, 931 (2009); R.C. Dewar, A. Maritan, arXiv:1107.1088.

[26] A. C. P. Rosa Jr. et al. Physica A 392, 6079-6083 (2013)

[27] M. Visser. JHEP 10 140, (2011).

[28] T. Wang. Phys. Rev. D 81 104045, (2010).

[29] S. H. Hendi and A. Sheykhi. Int. J. Theor. Phys 51 1125, (2012).

[30] M. Rocca, A. Plastino, G.L. Ferri: Physica A 393, 244 (2014)

[31] I. S. Gradshteyn and I. M. Rizhik: "Table of Integrals Series and Products". Academic Press (1965). 